\title{
Aggregate Interference in ISM Band: WBANs need cognitivity?
}

\author{
Lorenzo Mucchi $^{1}$, Alessio Carpini ${ }^{1}$ \\ ${ }^{1}$ Dept. of Information Engineering, University of Florence, Italy, lorenzo.mucchi@unifi.it
}

\begin{abstract}
Estimating the aggregate interference is required in order to predict the performance of a wireless system in its working environment. Body Area Networks (BANs) for healthcare applications are becoming a reality, allowing patients to be monitored continuously without forcing them to stay in bed or in hospital. The increasing number of wireless medical devices makes the ISM (Industrial, Scientific and Medical) band particularly crowded. If new smart BANs have to correctly operate in hospital, coexistence with the existing wireless devices must be accurately investigated, starting with studying the interference in the operating frequency band. In this paper we propose an interference model based on real measurements carried out in the emergency ward of a modern city hospital, as well as in home and office environments, which are the three main locations where a patient monitored by a WBAN spends the most of the time. The measurements are used to model the probability density function of the aggregate interference as well as the occupancy of the channel.
\end{abstract}

Keywords-interference modelling, real measurements, wireless communications, ISM band.

\section{INTRODUCTION}

Wireless communication system designers are commonly interested in basic questions like what is the maximum distance for which the wireless channel is suitable for communications or what are the best locations in the environment for the access points (transmitters). The simplest approach towards answering the above questions is by comparing two things: a) the dynamic range of the system in hand, and b) the electromagnetic waves propagation loss. The dynamic range is a characteristic of the system that is well known to the designer. It determines the maximum power loss that is allowed between transmitter and receiver, while still maintaining an effective communication link. The main characteristics that determine the dynamic range are the transmission power and receiver sensitivity. Although the theoretical laws of physics describe very accurately the different aspects of electromagnetic wave propagation, it is vital to understand that the complexity of real-life environments makes the actual propagation loss very difficult to predict. Most applications in today's short range RF scene operate in environments that are far from the ideal freespace scenario. In particular, modern city hospitals are a very harsh environment for wireless communications. Nowadays the wireless devices which make use of the ISM (Industrial, Scientific and Medical) band $\left(B_{s}=[2.399,2.485] \mathrm{GHz}\right)$ are very common. This increasing number of ISM devices, together with the extremely widespread adoption of Wi-Fi devices, can lead to a high level of interference in a typical modern city hospital [1]-[6]. This interference can be highly destructive, resulting in an alteration of the received power compared to the ideal free-space propagation. Early attempt to characterize the electromagnetic environment in hospitals dates back more than 30 years. In [7] the electromagnetic environment from $14 \mathrm{kHz}$ up to $1 \mathrm{GHz}$ in a hospital was assessed. In this paper, emission limits for narrowband and broadband signals were first recommended for devices used in hospitals. In 1997, the first 24-hour measurements showing temporal dependencies of the measured field within a hospital for frequencies up to $1 \mathrm{GHz}$ were described [8]. In 2003 the electromagnetic field within the ISM band ([2.40 - 2.4835] $\mathrm{GHz}$ ) was evaluated [9]. In [10] both short-term and long-term field strength measurements were carried out in the frequency range from $9 \mathrm{kHz}$ up to $10 \mathrm{GHz}$. Only electromagnetic emissions were collected in these field measurements.

In order to better define the performance of wireless medical devices operating in environments where an hypothetic patient which is monitored by a WBAN uses to stend the most of the time of a day, i.e., home, office and hospital, we carried out real-life measurements in a typical city hospital, home and office in order to model the interference behaviour. Concerning the hospital, the interference power levels have been recorded for 5 hours by using a spectrum analyzer in several locations of the emergency ward of the city Hospital "San Giuseppe" in Empoli, a medium-size town $20 \mathrm{~km}$ west of Florence, Italy. In the other environments, home and office, only one location is measured but a longer time, one week. The main parameters, such as the first and second order statistics, to characterize the probability density function (PDF) of the interference fluctuations are derived based on those measurements.

The evaluation of the amount of interference can give us a hint on how much interference is typical in the most used environments for persons wearing WBANs. This can also give us a hint on whether more cognitive smartness (sense, elaborate and decide/change) is needed in the WBAN terminals or not, in order to face the interference and enhance the quality of transmission of very sensitive data.

\section{MEASUREMENTS SETTINGS}

The measurements have been carried out in three environments: hospital, office and home.

\section{A. Hospital}

The field trials have been carried out in the emergency ward of a modern city hospital, namely the Hospital "San 
Giuseppe" in Empoli, a medium-size town $20 \mathrm{~km}$ west of Florence, Italy. The hospital ensemble includes four buildings and a surface of 43402 square meters, totally. The hospital has 24 wards, 413 beds, 8 operating rooms and 2 birthing rooms. Each recovery bed has a personal computer with Wi-Fi access for the entertainment of the patient. The hospital has also been equipped with the latest medical devices, most of which make use of wireless connection for transferring of medical data of the patient directly to the server and updating of the health history record.

All the measurements were taken during the busiest time of the day; medics and patients were moving around in the building. The emergency ward can be seen as a "worst case" of the hospital, since it normally includes all the medical devices which can radiate. The interference was evaluated by collecting data without probing signal, in the corridors and in the rooms (Fig. 1), over the entire ISM band, specifically the band $B_{s}=[2.399,2.4835] \mathrm{GHz}$. A spectrum analyzer (SA) by RF Explorer was used to measure the received power (in $\mathrm{dBm})$. The main parameters of the SA are the following: freq. range $2350-2700 \mathrm{MHz}$, freq. resolution $1 \mathrm{KHz}$, average noise level $-115 \mathrm{dBm}$ and amplitude resolution $0.5 \mathrm{dBm}$.

In Fig. 1 the map of the emergency ward is shown. The coloured points represent the locations where the interference was measured. The functionalities of the different zones are the following:

- Zone 1 is used for various types of ambulatory care units.

- Zone 2 is used for bedridden patients, with various health problems from acute diseases to chronic conditions.

- Zone 3 is used for various radiological analyses: X-ray, tomography, MRI, ultrasound, etc. Note that the room 21 in Zone 3 is for the extremely urgent operations (intensive care and surgery).

\section{B. Office and Home}

The measurements in the office and home environments have been carried out with the same device and setting of the ones described in the previous section. The office environment, as well as the home, is a typical city modern office, with several internal WiFi access points and surrounded by other offices and houses. The city has also a WiFi free access network for public use which is received in the office. The home environment has similar characterization apart from the public $\mathrm{WiFi}$, which is not present.

\section{RESULTS}

\section{A. Hospital}

In this section the results of the test fields will be discussed. The measurement results will be shown for Zones 1 and 3 only, for lack of space. Figs. 2-3 illustrate the interference power in the ISM band as a function of time and frequency for Zone 1 and Zone 3, respectively. It is evident that the profile of the interference power varies significantly as the receiver location changes, either across different zones or within the same zone.

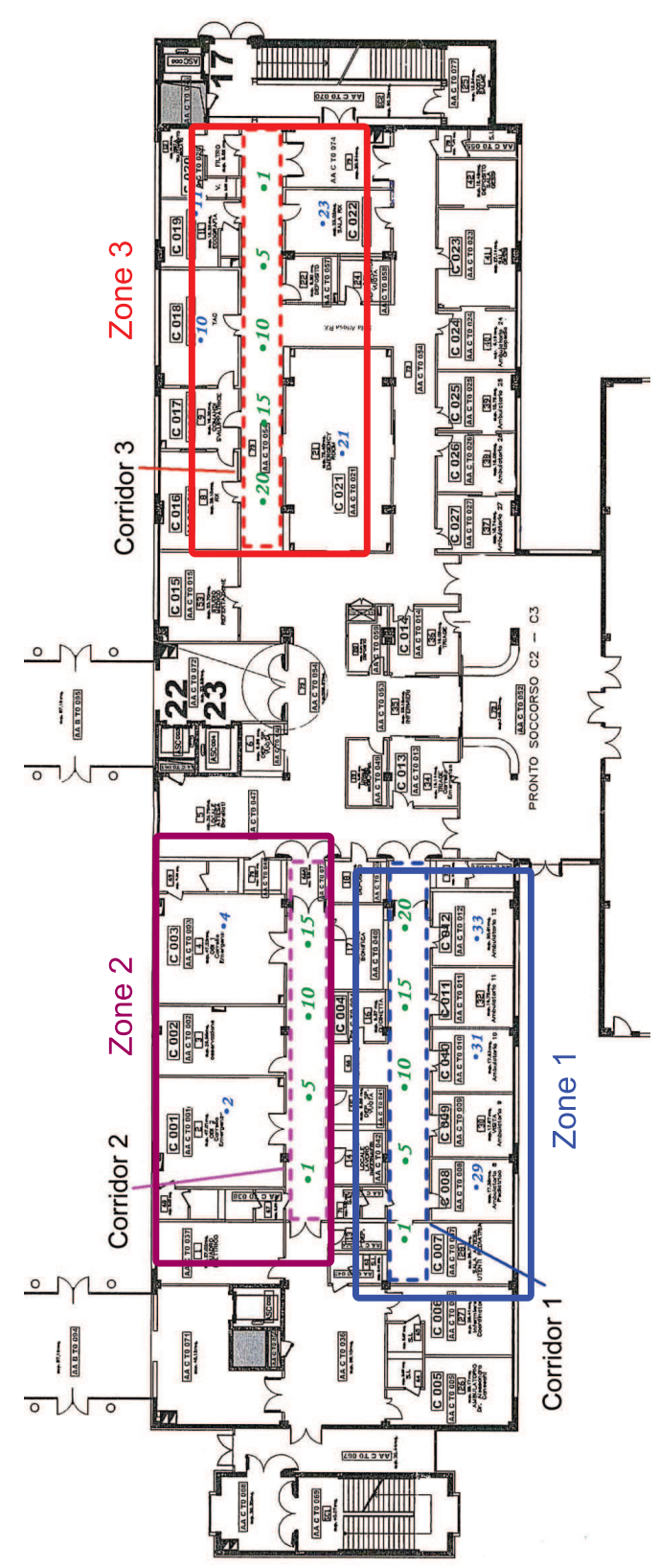

Fig. 1. The map of the emergency ward of the hospital. The corridors are highlighted by dashed lines. The green and blue points represent the locations where the measurements were taken.

More specifically, the following observations can be made. First, there is less interference in Rooms 10 and 23 in Fig. 3 as these are devoted to radiological operations and thus the walls are very thick, blocking electromagnetic waves. Rooms 31 and 33 in Fig. 2 show more interference, in some subbands with "impulsive" behaviour (interference higher than noise level only for a short time). In particular, Rooms 29 and 31 are close to an external door which is remotely controlled by a signal in the ISM band. Corridors of Zone 1 (Location 5) show higher interference compared to Zone 3 (Location 15) but not in all sub-bands. Corridor 3 shows interference more uniformly distributed over the frequencies.

Figs. 4-5 show the occupancy of the frequencies of the ISM 


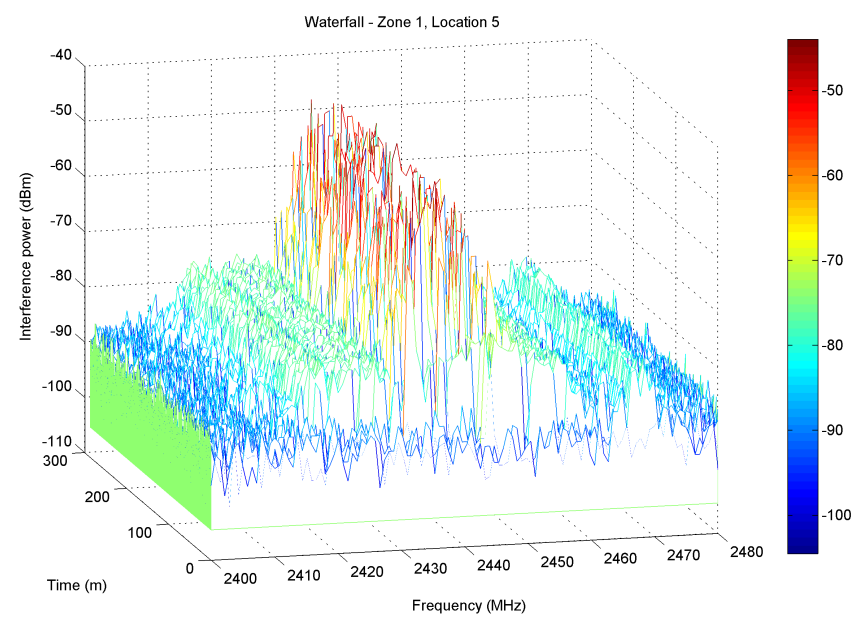

(a) Corridor 1, Location 5

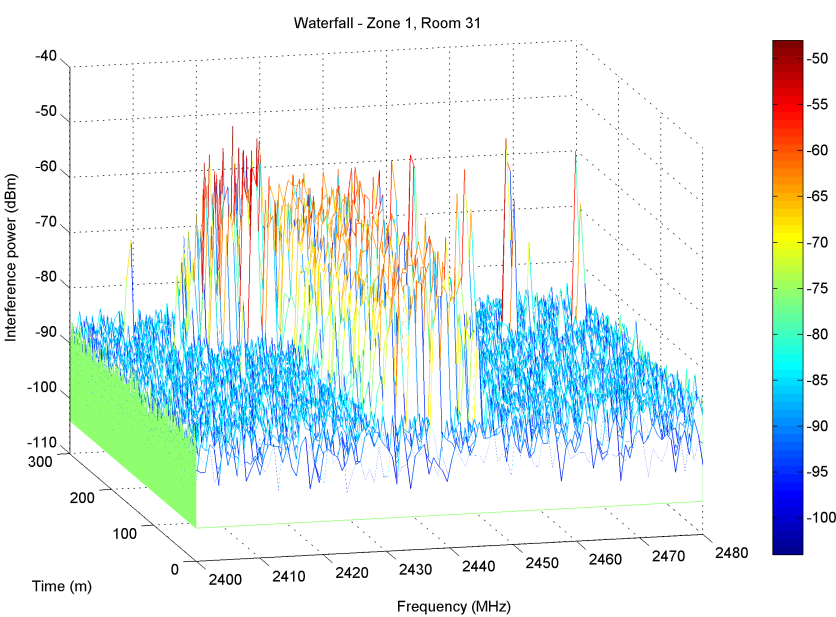

(b) Room 31

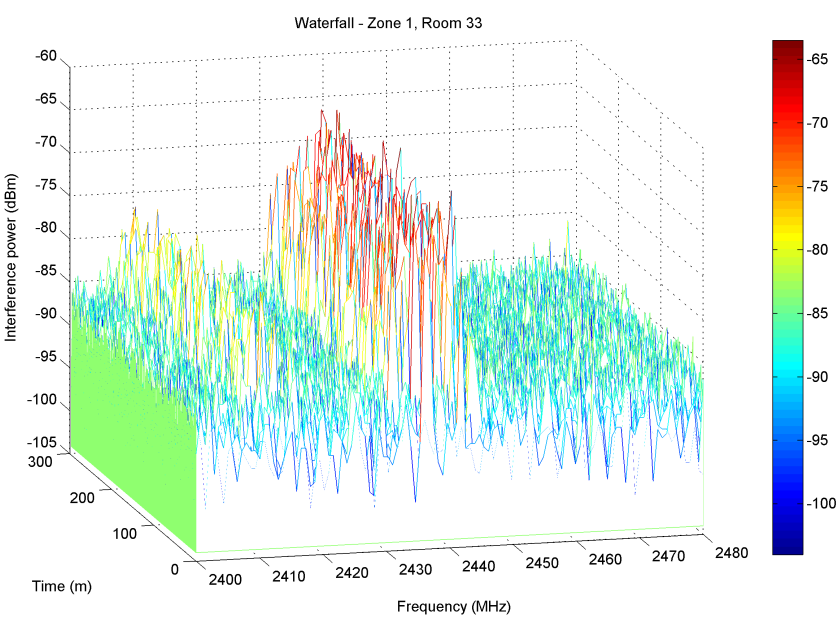

(c) Room 33

Fig. 2. Interference power ( $\mathrm{z}$-axis, $\mathrm{dBm})$ in the ISM band as a function of Time (y-axis, minutes) and Frequency (x-axis, MHz) for Zone 1.

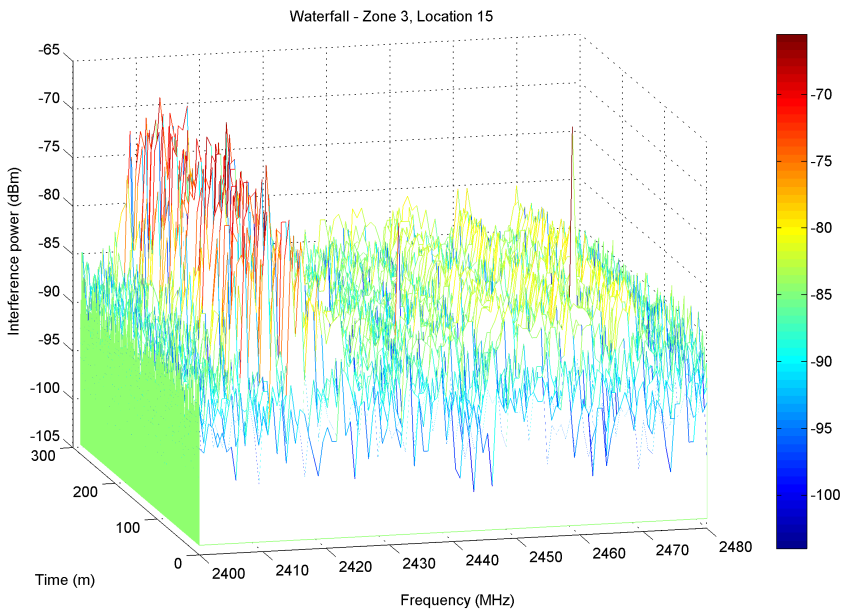

(a) Corridor 3, Location 15

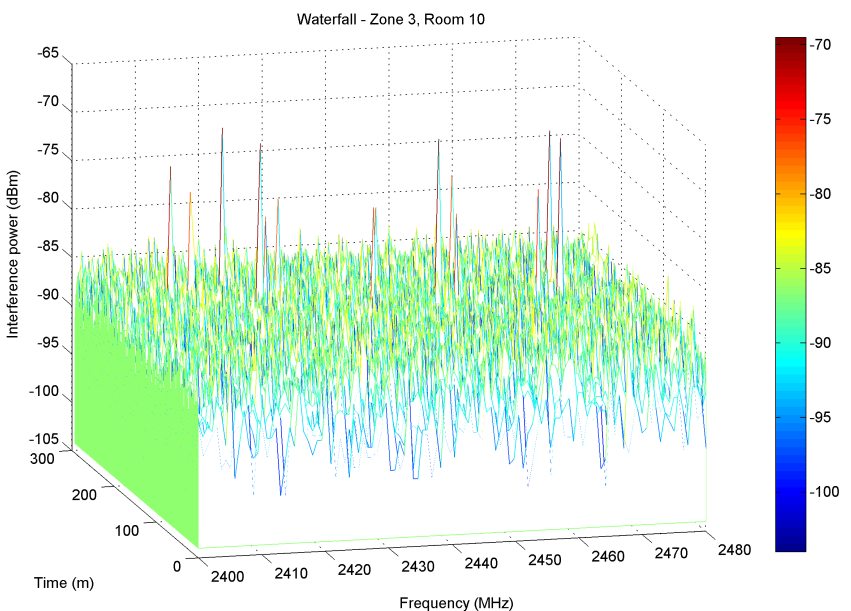

(b) Room 10

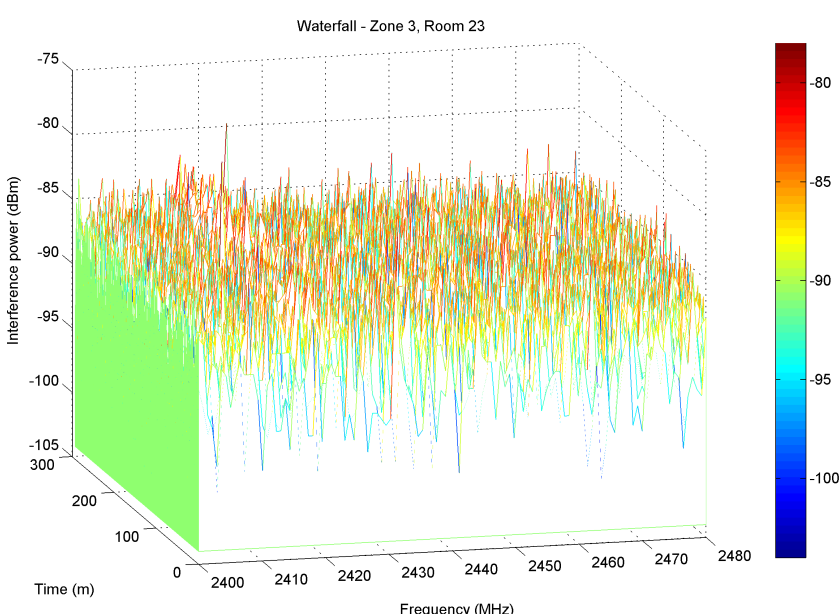

(c) Room 23

Fig. 3. Interference power (z-axis, $\mathrm{dBm}$ ) in the ISM band as a function of Time (y-axis, minutes) and Frequency (x-axis, MHz) for Zone 3. 
band over time, i.e., the percentage of time that the received amplitude keeps $20 \mathrm{~dB}$ over the noise level. The average noise level (ANL) was measured to be $-100 \mathrm{dBm}$.

The interference behaviour can be very different from one location to another, depending on the type of ward, the type of room, etc. In any case, the percentage of occupancy is not so high, even in the worst location. Thus, we can conclude that wireless body sensors and body area networks can be used without particular mechanisms for interference rejection. The percentiles show that only a small amount of interference power samples lay over the threshold, e.g., in Room 31 only $1 \%$ of the received samples lay over $-80 \mathrm{dBm}$.

We defined two thresholds in order to decide if the channel can be said occupied or not. The first is derived as the minimum power at the receiver in order to have a probability of error $P_{e}$ of $10^{-3}$ at least, in an ideal AWGN channel and antipodal symbols transmission. A $P_{e}=10^{-3}$ implies a high quality link, taking into account that these are uncoded bits. Using a binary antipodal signalling in AWGN channel, a signal-to-noise ratio (SNR) of $7 \mathrm{~dB}$ is needed at the receiver in order to guarantee $P_{e}=10^{-3}$ [11]. With a value of $A N L=-100 \mathrm{dBm}$, we can derive that a minimum power of $-63 \mathrm{dBm}$ is needed at the receiver, thus the first threshold is set to $-63 \mathrm{dBm}$.

The second threshold we set to decide if a channel is occupied or not is $-80 \mathrm{dBm}$. This value takes into account the fading margin compared to the previous threshold. This value came from another experiment [4] where the quality of a wireless ECG signal was measured in different places of the same hospital ward. The experiment highlighted as a received power lower than $-80 \mathrm{dBm}$ leaded to unacceptable quality of ECG signal (the number of lost packets was too high). The occupancy figures 4-5 show the percentiles of the interference power samples, compared to the two thresholds mentioned above.

Using the measurements we have investigated the best fitting probability distribution function (PDF) of the received power samples. We have tried 16 different distributions for all the locations where we have collected data. In particular, the distributions are: Beta, Birnbaum-Saunders, Exponential, Extreme value, Gamma, Generalized extreme value, Generalized Pareto, Inverse Gaussian, Logistic, Log-logistic, Lognormal, Nakagami, Normal, Rayleigh, Rician, $t$ location-scale and Weibull. The best fitting distribution has been derived by using a specific Matlab script. The Matlab script finds the most fitting distribution by using the maximum likelihood criteria. Two exceptions are the normal and lognormal distributions with uncensored data. For the uncensored normal distribution, the estimated value of the sigma parameter (standard deviation) is the square root of the unbiased estimate of the variance. For the uncensored lognormal distribution, the estimated value of the sigma parameter is the square root of the unbiased estimate of the variance of the $\log$ of the data.

The results show that when the interference is more prominent like in Zone 1 the best-fit PDF is the $t$ location-scale, while if the interference is lower like in Zone 3 then the bestfit PDF changes to the generalized extreme value or normal.

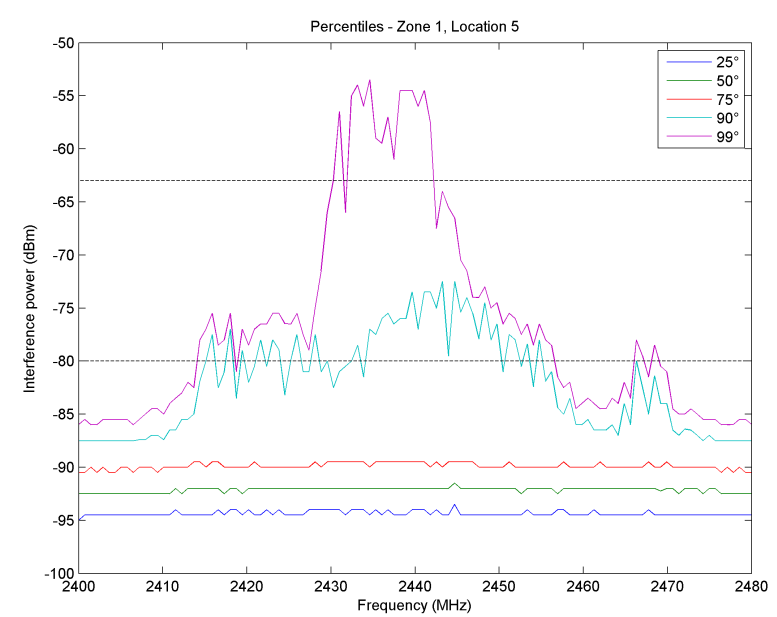

(a) Corridor 1, Location 5

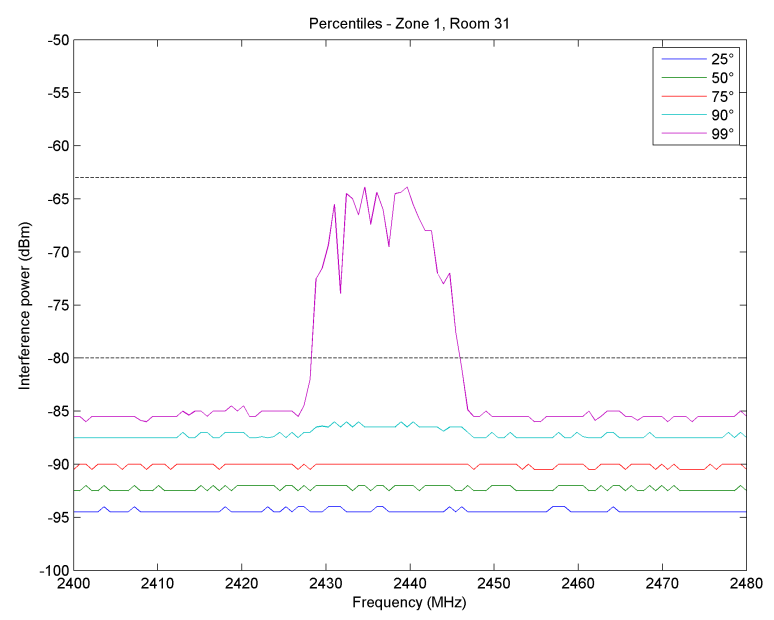

(b) Room 31

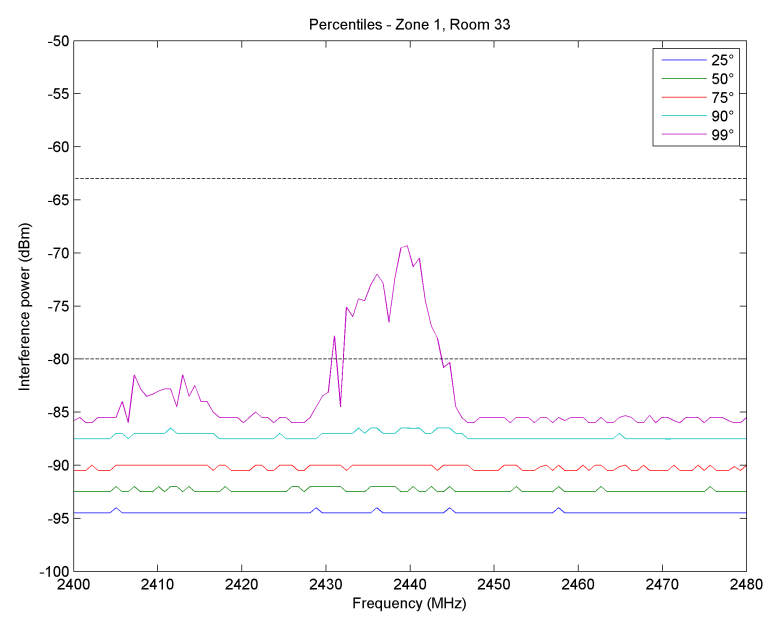

(c) Room 33

Fig. 4. Percentiles of the interference power samples in the ISM band for Zone 1. 


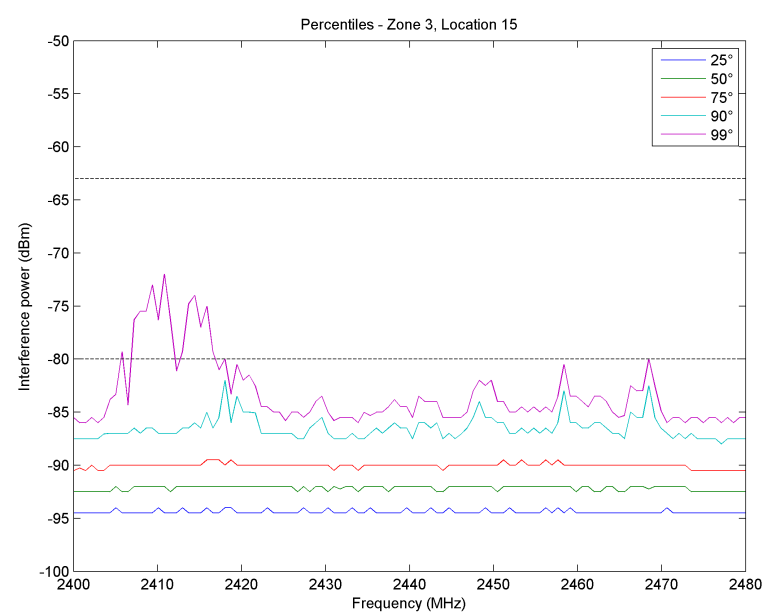

(a) Corridor 3, Location 15

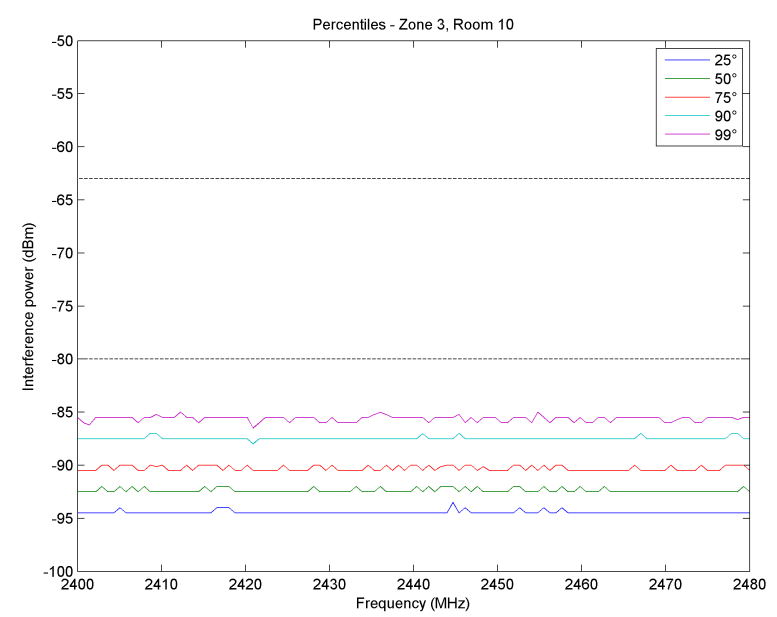

(b) Room 10

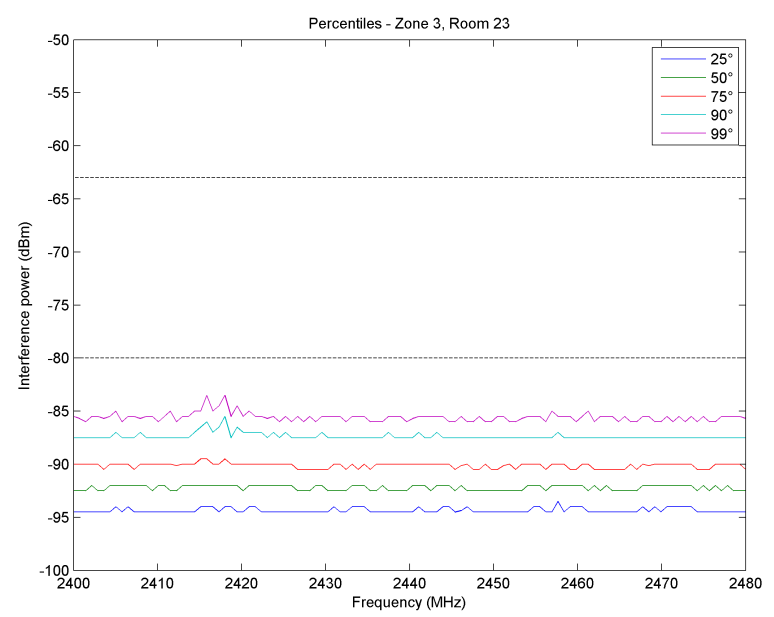

(c) Room 23
The $t$ location-scale distribution has a density function of

$$
p(x \mid \nu, \mu, \sigma)=\frac{\Gamma\left(\frac{\nu+1}{2}\right)}{\sigma \sqrt{\pi \nu} \Gamma\left(\frac{\nu}{2}\right)}\left(\frac{\nu+\left(\frac{x-\mu}{\sigma}\right)}{\nu}\right)^{-\frac{\nu+1}{2}}
$$

where $\Gamma(t)=\int_{0}^{\infty} x^{t-1} e^{-x} d x$ is the Gamma function, $\mu$ is the location parameter, $\sigma>0$ is the scale parameter and $\nu>0$ is the shape parameter. If $x$ has a $t$ locationscale distribution, with parameters $\mu, \sigma$ and $\nu$, then $\frac{x-\mu}{\sigma}$ has a Student's $t$ distribution with $\nu$ degrees of freedom. The $t$ location-scale distribution is useful for modeling data distributions with heavier tails (more prone to outliers) than the normal distribution. It approaches the normal distribution as $\nu$ approaches infinity, and smaller values of $\nu$ yield heavier tails.

The PDF for the generalized extreme value distribution with location parameter $\mu$, scale parameter $\sigma$, and shape parameter $k \neq 0$ is

$$
p(x \mid k, \mu, \sigma)=\frac{1}{\sigma} e^{-\left(1+k \frac{x-\mu}{\sigma}\right)^{-\frac{1}{k}}}\left(1+k \frac{x-\mu}{\sigma}\right)^{-1-\frac{1}{k}}
$$

for $1+k \frac{x-\mu}{\sigma}>0$ and $k>0$. The case $k>0$ corresponds to the extreme value Type II distribution, also known as Frechet distribution, while $k<0$ corresponds to the Type III, also known as Wiebull distribution and $k=0$ corresponds to the Type I, also known as Gambel distribution.

The main parameters of each of the best-fit distributions, e.g., $(\mu, \sigma, \nu)$ for the $t$ location-scale and $(k, \mu, \sigma)$ for the generalized extreme value, for each frequency of the ISM band and for each location have been derived. These parameters are not shown here due to the lack of space. Using these parameters it is possible to build the correct distribution of the interference in the specific environment of the hospital. The interference distribution can be used to predict the quality of the received signal and thus design the main parameters of the wireless body area networks for a proper use inside the hospital.

\section{B. Office and Home}

The office and home measurements are reported in Figs. 67. In particular, the percentage of occupancy of the frequencies in the ISM band as well as the interference power as a function of time and frequency are depicted for both the office and home environment. The definition of occupancy is in Sec. III-A. The occupancy is here highlighted by using the percentiles. A percentile indicates the value below which a given percentage of observations in a group of observations fall. Although the Office (Fig. 7) could seem at first look as the worst environment, a deeper look reveals as only a small amount of samples (1\% of the overall 1-week long measured samples) show a magnitude greater than the $-80 \mathrm{dBm}$ threshold.

Analyzing the results, we can deduce that there is a correlation between the occupancy and the estimated distribution of the channel. In fact,

- For frequencies poorly utilized, the best fitting PDF of the interference samples over time is a normal or a generalized extreme value distribution;

Fig. 5. Percentiles of the interference power samples in the ISM band for 


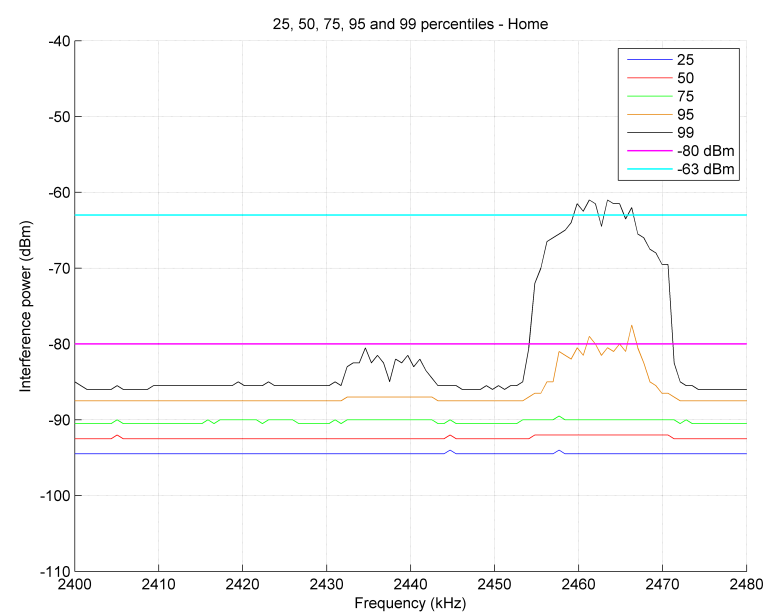

(a) Percentiles of the interference power samples in the ISM band.

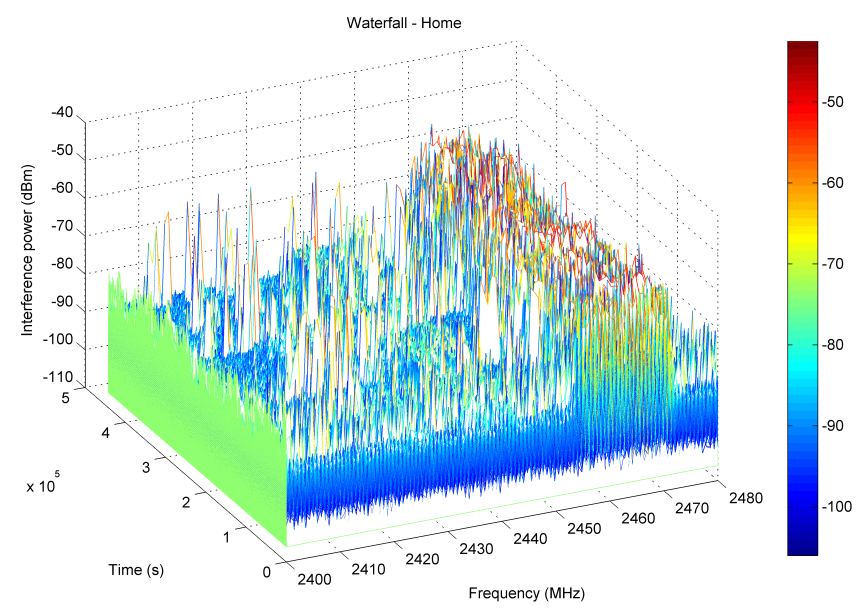

(b) Interference power (z-axis, $\mathrm{dBm}$ ) in the ISM band as a function of Time (y-axis, sec) and Frequency (x-axis, MHz).

Fig. 6. Home environment.

- For frequencies with high percentage of occupancy, the best fitting PDF of the interference samples is a $t$ location-scale distribution.

\section{CONCLUSION}

In this paper we have presented a measurement campaign aiming to collect the interference power levels in a modern city hospital, office and home. The data have been collected in different locations of the emergency ward during a typical busy day. For the home and office environments the measurements lasted for one week. The data have been used to show the typical interference levels in the ISM band. The best fitting distributions have been derived for each frequency and location. For each distribution the main parameters of the best-fit PDF have been derived. Thus the interference behaviour can

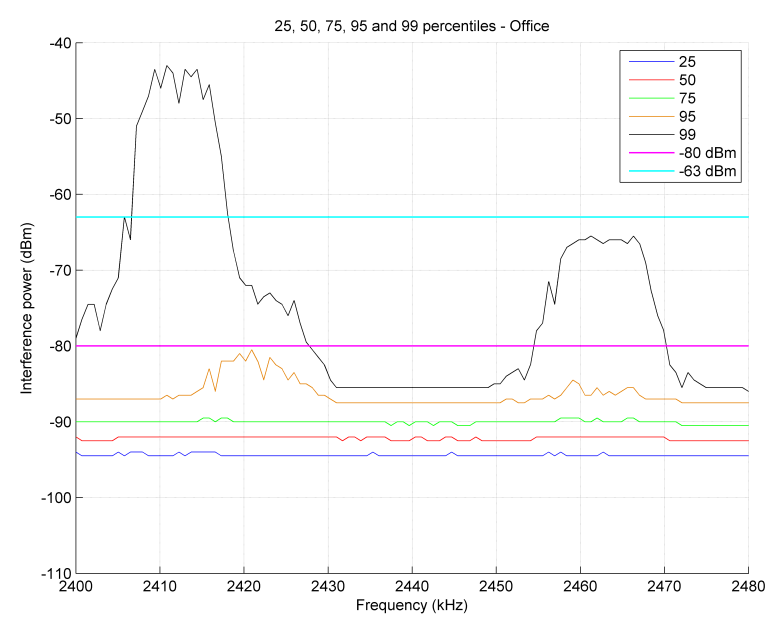

(a) Percentiles of the interference power samples in the ISM band.

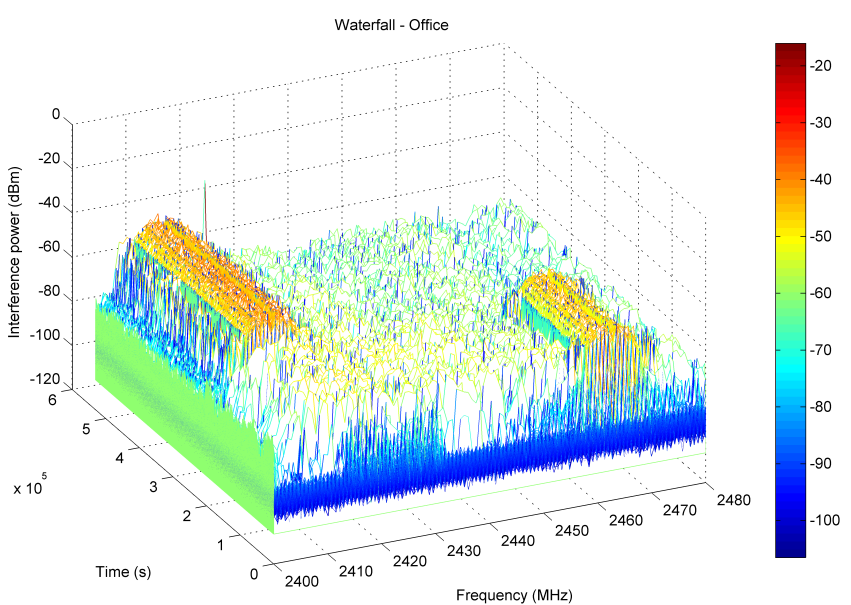

(b) Interference power (z-axis, $\mathrm{dBm}$ ) in the ISM band as a function of Time (y-axis, sec) and Frequency (x-axis, MHz).

Fig. 7. Office environment.

be reproduced in order to test and design the use of wireless body area networks in the main locations a monitored patient spends the most of the time (home, office, hospital).

This preliminary research leaded us to a preliminary conclusion: the amount of interference is not dramatic in the hospital, home and office environments to justify an added smartness (and thus complexity) in the transceivers of WBANs. Of course, this is true currently in Italy, other countries could experience different average levels of interference in the ISM band in those environments.

\section{ACKNOWLEDGMENT}

This research was partially conducted under the project "ASSO", funded by the Tuscany Region, Italy (POR CreO FESR 2007-2013, Linee di Attivita' 1.5.a e 1.6, Bando Unico 
RS 2012), and under the project "CoNHealth", FP7 Marie Curie People Action.

\section{REFERENCES}

[1] L. Mucchi, F. Trippi, Wireless Real-Time System for measuring performance and health in sports, 2nd International Workshop on Future Wellness and Medical ICT Systems (FEELIT), in conjunction with WPMC 2011, Brest, France, 3-6 Oct. 2011.

[2] L. Mucchi, F. Trippi, A. Carpini, M. Amato, R. Cozzani, Performance of Wireless Real-Time System for ECG Monitoring in Hospitals, International Symposium on Medical Information and Communication Technology (ISMICT), 26-29 March 2012, San Diego, CA.

[3] L. Mucchi, F. Trippi, A. Carpini, Ultra Wide Band Real-Time Location System for Cinematic Survey in Sports, ISABEL Conference, 7-10 Nov. 2010, Rome, Italy.

[4] L. Mucchi, F. Trippi, A. Carpini, M. Amato, Performance of Wireless ECG Monitoring in Hospitals in presence of interference, 7th International Conference on Body Area Networks, Workshop on Ultra Wide Band for Body Area Networking (UWBAN), Oslo, 24-26 Sept. 2012.

[5] L. Mucchi, F. Trippi, A. Carpini, M. Amato, R. Cozzani, Wearable Wireless ECG System for Personal Health-care Application, ISMICT 2013, Tokyo, Japan, 6-8 March 2013.

[6] L. Mucchi, Measurements campaign for interference estimation and performance assessment of a wireless ECG remote monitoring prototype in a city hospital, VTC Fall 2013, 2-5 Sept. 2013, Las Vegas, USA.

[7] U. A. Frank and R. Londner, The hospital electromagnetic interference environment, Journal of the association for advancement of medical instrumentation, vol. 5, no. 4, pp. 246-254, 1971.

[8] P. Vlach, C. Liu-Hinz, B. Segal, B. Skulic, T. Pavlasek, The electromagnetic environment due to portable sources in a typical hospital room, IEEE 17th Annual Engineering in Medicine and Biology Society Conference, vol. 1, pp. 683-684, 1995.

[9] S. Krishnamoorthy, J. Reed, C. Anderson, M. Robert, and S. Srikanteswara, Characterization of the $2.4 \mathrm{GHz}$ ISM band electromagnetic interference in a hospital environment, IEEE 25th Annual Int. Conf. on Engineering in Medicine and Biology, vol. 4, pp. 3245-3248, 2003.

[10] O. Lauer, M. Riederer et al., Characterization of the electromagnetic environment in a hospital, Electromagnetic Compatibility and 19th International Zurich Symposium on Electromagnetic Compatibility, AsiaPacific Symposium on, 2008, pp. 474-477.

[11] J. Proakis, M. Salehi, Digital Communications, McGraw-Hill, 5th Edition, 2007. 